\title{
INFLUENCE OF GENDER AND OBESITY ON ANALGESIC MODULATION OF TRAMADOL IN RATS
}

\author{
SHAKTA MANI SATYAM ${ }^{1 *}$, LAXMINARAYANA KURADY BAIRY², VASUDHA DEVI ${ }^{1}$
}

${ }^{1}$ Department of Pharmacology, Melaka Manipal Medical College, Manipal Campus, Manipal Academy of Higher Education, Manipal, Karnataka, India. ${ }^{2}$ Department of Pharmacology, RAK College of Medical Sciences, RAK Medical and Health Sciences University, North Ras Al Khaimah, United Arab Emirates. Email: smsatyam21@gmail.com

Received: 10 April 2018, Revised and Accepted: 04 May 2018

\begin{abstract}
Objective: The objective of this study was to investigate the influence of gender and obesity on analgesic modulation of tramadol in Wistar rats.

Methods: This study was carried out in two sets of experiments. In Set I experiment - 48 rats (body weight $\leq 150$ g), 24 each male and female rats were randomly divided into two groups (n=6/group) (Group I - Control; 0.9\% NaCl; $1 \mathrm{ml} / \mathrm{kg} / \mathrm{day}$ i.p. and Group II - Tramadol $10 \mathrm{mg} / \mathrm{kg} / \mathrm{day}$ i.p.) for each nociception model - plantar test and acetic acid-induced writhing test. The treatment duration was of 5 days. On the last day of treatment (i.e., on the $5^{\text {th }}$ day), paw withdrawal latency (PWL) was assessed using plantar test, and writhing movements were observed following administration of $0.8 \%$ acetic acid; $10 \mathrm{ml} / \mathrm{kg}$ i.p. Set II experiment was repeated like Set I experiment among rest 48 high-fat diet-fed rats (body weight $\geq 300$ g).

Results: For both males and females, PWL was significantly decreased $(\mathrm{p}<0.05)$ in obese control groups compared to lean control groups. A number of writhing movements were significantly increased ( $p<0.01$ for males and $p<0.001$ for females) in obese control groups compared to lean control groups. In tramadol-treated obese rats, PWL was significantly decreased ( $\mathrm{p}<0.01$ for males and $\mathrm{p}<0.05$ for females), and number of writhing movements were significantly increased ( $\mathrm{p}<0.01$ for both males and females) in comparison with the tramadol-treated lean rats.
\end{abstract}

Conclusion: The present study revealed that obese female rats experience more pain sensation to noxious stimuli compared to lean male rats and also the analgesic effect of tramadol is more pronounced in lean male rats compared to obese female rats.

Keywords: Pain threshold, Sex hormone, Opioids, Body weight, Writhing, Plantar, Acetic acid.

(C) 2018 The Authors. Published by Innovare Academic Sciences Pvt Ltd. This is an open access article under the CC BY license (http://creativecommons. org/licenses/by/4. 0/) DOI: http://dx.doi.org/10.22159/ajpcr.2018.v11i8.26624

\section{INTRODUCTION}

According to the International Association for the Study of Pain (IASP), pain is an unpleasant sensory and emotional experience arising from actual or potential tissue damage or described in terms of such damage [1]. Sex differences in pain and its control have long been a debated issue for scientists and health-care providers hoping to optimize pain treatment for the individual. The current scenario toward evidence-based medicine has further highlighted this issue. In the year 2007, the sex, gender, and pain special interest group of the IASP issued a consensus paper highlighting the need for inclusion of both males and females in pre-clinical and clinical studies on pain and its management [2]. In one of the randomized clinical trials, females have been shown to experience greater intensity of post-operative pain and less tolerance to pain compared to men [3].

Rosseland and Stubhaug stated gender difference in response to opioids, but their findings are inconsistent [4]. Some studies suggest that female patients experience greater analgesic efficacy compared to males following administration of mixed opioid agonist-antagonists, whereas other studies with morphine demonstrate that women require a higher drug dose compared to males to achieve analgesia of the same degree [3-5].

Several studies have positively correlated the experience of pain with an increase in body mass index (BMI) $[6,7]$. The causal relationship between the two remains unclear: It is not known whether obesity causes chronic pain, chronic pain causes obesity, or some other factors cause both concurrently. Obesity is hypothesized to lead to pain because of excess mechanical stresses and its proinflammatory state.

Obesity can affect pain threshold, emotional mood, and quality of life. Differences in pain thresholds and emotional mood may have implications for pain and depression management. Differences in pain thresholds may have implications for pain management, as they may account in part for the variability in analgesic requirements between individuals. In obese patients, pain thresholds would make it possible to predict the need for prescriptions of drugs with a narrow therapeutic margin, such as morphine [8]. McKendall and Haier conducted a study based on the hypothesis claiming that analgesic opiates are increased in obesity [9]. They applied a constant pressure of approximately three pounds to the tip of the thumb on 56 obese and non-obese patients to measure the time interval until the first sensation of pain. However, contrary to the expectations, the obese patients were found to be more sensitive to pain. Roane and Porter used tail pinch or tail flick pain stimulation tests and found that Zucker rats were more sensitive to pain [10]

There is need for further studies to investigate the influence of gender and obesity on pain threshold and analgesic modulation of opioids. This might help to modify the practice of prescribing analgesic medication according to gender and BMI. Tramadol is a synthetic, centrally acting analgesic agent with two distinct, synergistic mechanisms of action, and acting as both a weak opioid agonist and an inhibitor of monoamine neurotransmitter reuptake [11]. Earlier, we had reported the analgesic modulation of some analgesics in male and female Wistar rats [12]. Consequently, the aim of the present study was to investigate the effect of tramadol on pain threshold among male and female Wistar rats using plantar test (Hargreaves' method) and acetic acid-induced writhing test.

\section{MATERIALS AND METHODS}

Drugs and chemicals

Active pharmaceutical ingredient of tramadol was obtained from Sigma-Aldrich, Bangalore (India). Acetic acid and other chemicals were purchased from Merck Life Sciences Pvt. Ltd., Mumbai (India). 
Normal saline $(0.9 \%$ Sodium chloride) was purchased from pharmacy of Kasturba Hospital, Manipal, Karnataka (India).

\section{Animals}

A total of 96 Wistar rats (48 each male and female) weighing 100$150 \mathrm{~g}$ were housed in separate polypropylene cages, maintained under standard conditions with temperature $\left(22-24^{\circ} \mathrm{C}\right), 12$-h light/12-h dark cycle, and relative air humidity $40-60 \%$. The animals were acclimatized to the laboratory conditions for 1 week before the start of the experiment. The animals were provided with a normal pellet diet (Amrit Feeds Ltd., Pune, India) and water ad libitum. The experimental protocol was approved by the institutional animal ethics committee (IAEC/KMC/41/2014), and experiments were conducted according to the ethical norms approved by Ministry of Social Justice and Empowerment, Government of India and Committee for the Purpose of Control and Supervision on Experiments on Animals guidelines.

\section{Experimental design}

A total of 96 Wistar rats were included in this study. The study was carried out in two sets of experiments. In Set I experiment - 48 lean rats (body weight 100-150 g), 24 each male and female rats were randomly divided into two groups (n=6/group) (Group I - control; $0.9 \% \mathrm{NaCl}$; $1 \mathrm{ml} / \mathrm{kg} /$ day i.p. and Group II - tramadol $10 \mathrm{mg} / \mathrm{kg} /$ day i.p.) for each nociception model - plantar test and acetic acid-induced writhing test (Fig. 1). Set II experiment was repeated like Set I experiment among rest 48 high-fat diet-fed (Vanaspati dalda + Coconut oil; 3:1; $10 \mathrm{ml} / \mathrm{kg}$ / day for 90 days) obese rats (body weight $\geq 300$ g) (Fig. 2). The treatment duration of this study was 5 days. On the last day of treatment (i.e., on the $5^{\text {th }}$ day), 15 min after administration of normal saline/tramadol, paw withdrawal latency (PWL) was assessed using plantar test, and writhing movements were observed following administration of $0.8 \%$ acetic acid; $10 \mathrm{ml} / \mathrm{kg}$ i.p [12-14].

\section{High-fat diet-induced obesity model}

For Set II experiment, 48 Wistar rats (100-150 g, 24 males and 24 females) were housed as two rats/cage. In addition to the normal pellet diet, these animals were orally fed mixture of Vanaspati dalda + Coconut oil (3:1) - $10 \mathrm{ml} / \mathrm{kg} /$ day for 90 days. After 90 days, these rats attained body weight approximately $\geq 300 \mathrm{~g}$. Thereafter, Set II experiment was repeated like Set I experiment on these 48 obese (body weight $\geq 300 \mathrm{~g}$ ).

\section{Nociception models}

Plantar test (Hargreaves' method)

Thermal pain threshold to radiant heat (IR-90) was quantified using the paw withdrawal test (Fig. 3). Rats were placed in a Perspex enclosure, without restraint and a movable infrared radiant heat source placed directly under the plantar surface of the hind paw (Ugo Basile, Como, Italy). The PWL to radiant heat was defined as the time from onset of the radiant heat to the withdrawal of the rat hind paw. The cutoff time for PWL was $30 \mathrm{~s}$. Testing was alternated between hind paws and carried out at 3 min intervals. The average of three estimations was taken to yield mean PWL. Before any testing was carried out, rats could adjust to their environments for at least $10 \mathrm{~min}$.

\section{Acetic acid-induced writhing test}

Writhing movement was induced by administering an intraperitoneal injection of $0.8 \%$ acetic acid $(10 \mathrm{ml} / \mathrm{kg}), 15 \mathrm{~min}$ after the tramadol/ normal saline administration. After $10 \mathrm{~min}$ of acetic acid administration, the number of writhing movements such as abdominal constriction/ elongation of body/arching of back/hind limb extension/forelimb extension/trunk twisting (Fig. 4) was cumulatively counted over 20 min further for nociceptive evaluation [12-14].

\section{Statistical analysis}

Using Statistical Package for the Social Sciences (SPSS version 16.0; SPSS Inc., Chicago, USA), data were expressed as mean \pm standard

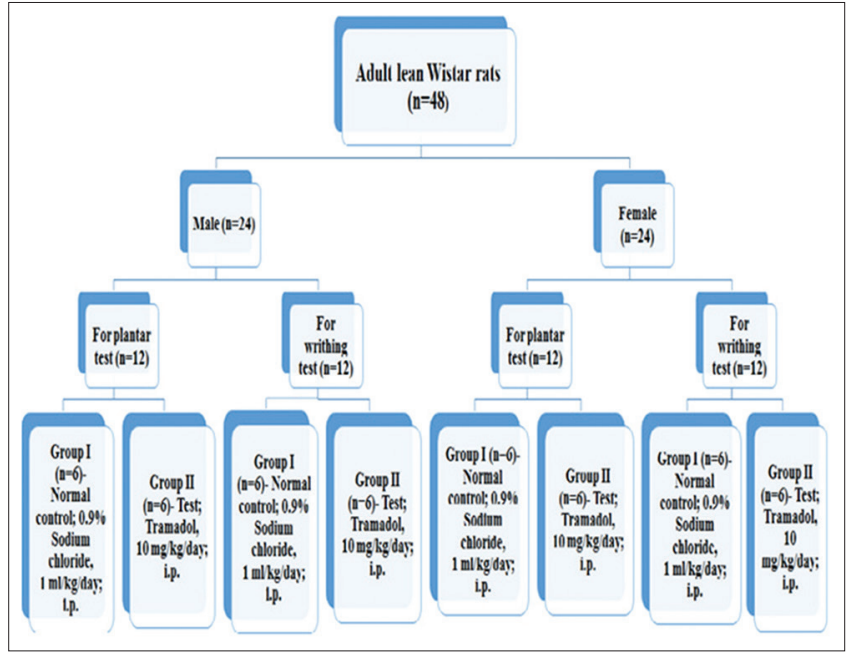

Fig. 1: Experimental design for lean Wistar rats (Set-I experiment)



Fig. 2: Experimental design for obese Wistar rats (Set-II experiment)

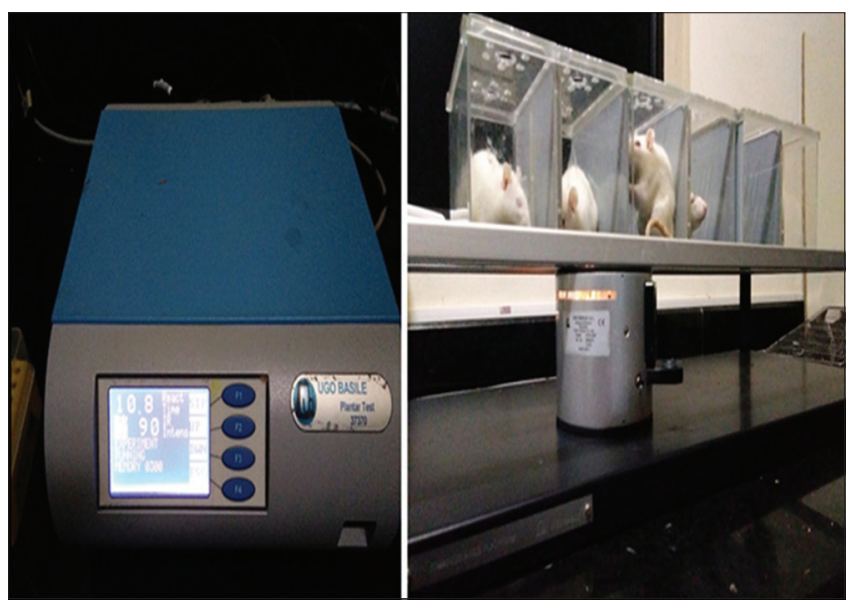

Fig. 3: Hargreaves apparatus for plantar test

deviation and analyzed by one-way analysis of variance followed by post hoc Tukey test. A level for $\mathrm{p} \leq 0.05$ was considered as statistically significant. 


\section{RESULTS}

For both lean and obese rats, PWL was significantly decreased $(\mathrm{p}<0.001)$ and number of writhing movements were significantly increased $(p<0.001)$ in female control groups compared to male control groups. Furthermore, in tramadol-treated female rats, PWL was significantly decreased ( $p<0.01$ for lean and $p<0.05$ for obese groups) and number of writhing movements were significantly increased ( $<<0.001$ for both lean and obese groups) in comparison with the tramadol-treated male rats (Figs. 5 and 6).

For both males and females, PWL was significantly decreased $(\mathrm{p}<0.05)$ in obese control groups compared to lean control groups. Number of writhing movements were significantly increased $(\mathrm{p}<0.01$ for males and $\mathrm{p}<0.001$ for females) in obese control groups compared to lean control groups. In tramadol-treated obese rats, PWL was significantly decreased ( $<<0.01$ for males and $p<0.05$ for females) and number of writhing movements were significantly increased $(\mathrm{p}<0.01$ for both males and females) in comparison with the tramadol-treated lean rats (Figs. 7 and 8).
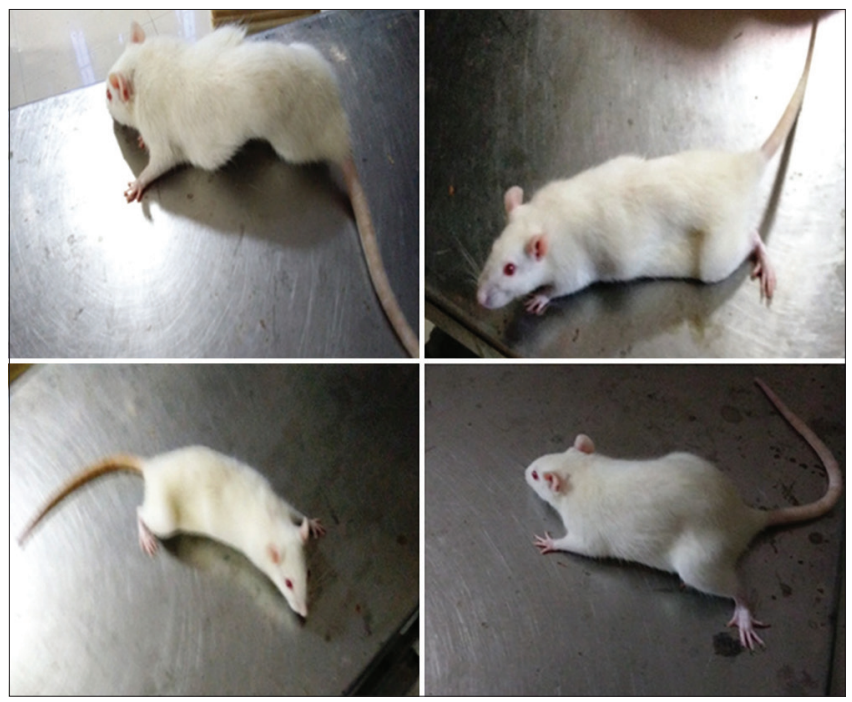

Fig. 4: Acetic acid-induced writhing movements

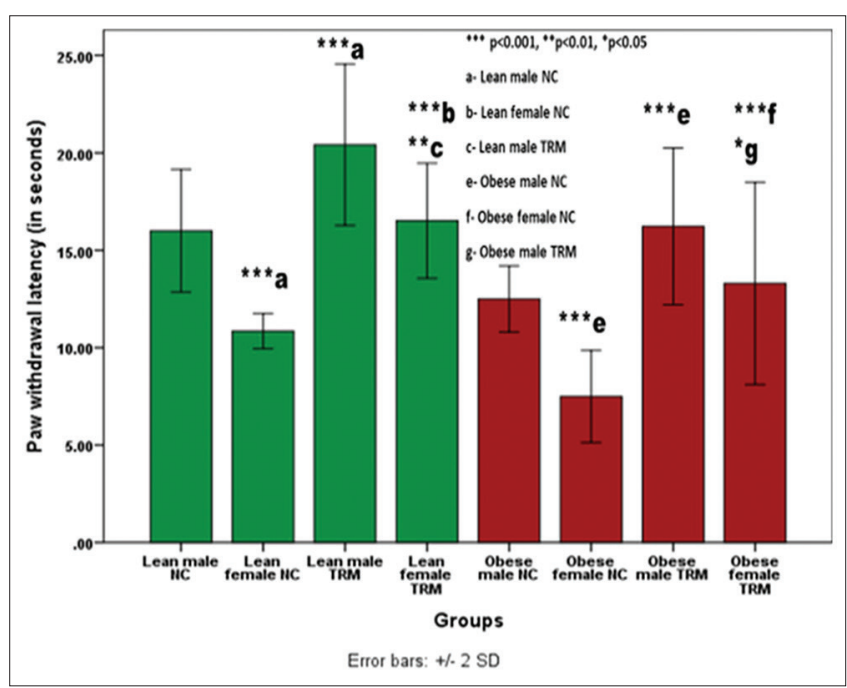

Fig. 5: Overall, comparison of paw withdrawal latency among both lean and obese male and female rats. $n=6$, number of rats in each group; NC: Normal control; TRM: Tramadol. Values are mentioned as mean. Error bars, \pm 2 standard deviation

\section{DISCUSSION}

The present study has demonstrated the influence of gender and obesity on analgesic modulation of tramadol in Wistar rats using plantar test and acetic acid-induced writhing test. This study has revealed the lower pain threshold in obese female rats than the lean male rats. In experimentally induced pain studies, the majority of results show that women are comparatively less tolerant and more sensitive to noxious stimulation than men [15-17]. Sex-related differences in pain may also reflect differences in the endogenous opioid system. The most studied of the endogenous pain modulatory systems is the endogenous opioid system, and sex differences in the functioning of this system could arise based on several different mechanisms. First, sex differences could result from differences in the distribution, expression, or sensitivity of opioid receptors in regions of the central nervous system involved in nociceptive processing. Sex-specific differences in gonadal hormones, genetic factors, central nervous system pain and pain



Fig. 6: Overall, comparison of number of writhing movements among both lean and obese male and female rats. $n=6$, number of rats in each group; NC: Normal control; TRM: Tramadol. Values are mentioned as mean. Error bars, \pm 2 standard deviation

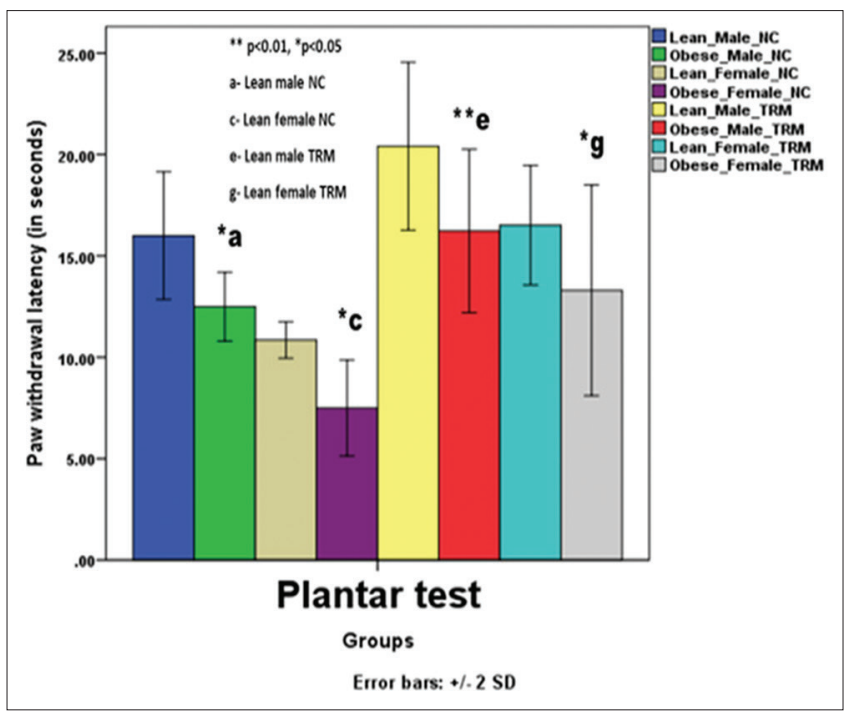

Fig. 7: Comparative analysis of paw withdrawal latency for lean versus obese rats. $n=6$, number of rats in each group; NC: Normal control; TRM: Tramadol. Values are mentioned as mean. Error bars, \pm 2 standard deviation 


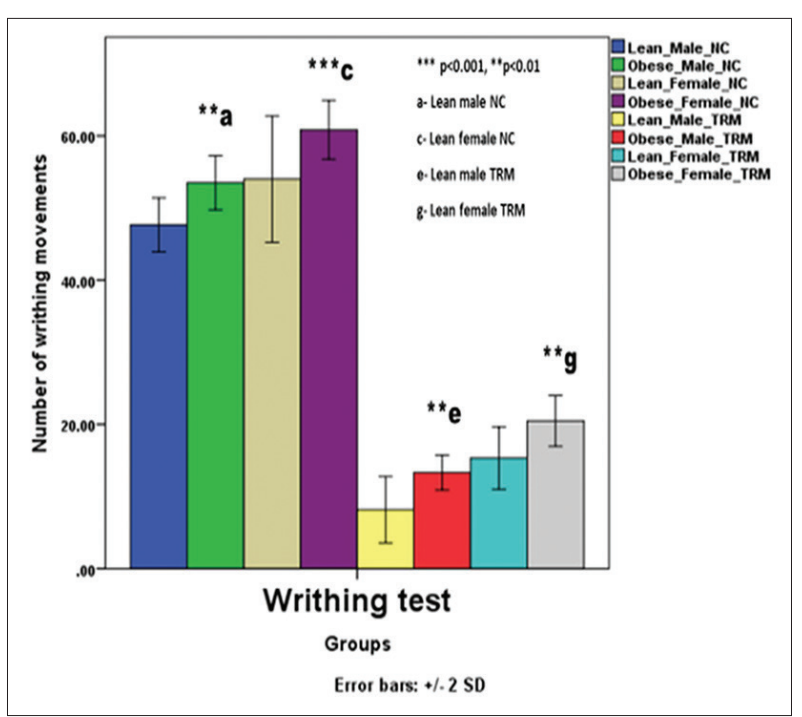

Fig. 8: Comparative analysis of number of writhing movements for lean versus obese rats. $n=6$, number of rats in each group; NC: Normal control; TRM: Tramadol. Values are mentioned as mean. Error bars, \pm 2 standard deviation

modulation circuitry, pharmacokinetic/pharmacodynamic factors, and psychosocial factors have been advanced as potential mechanisms for sex differences in opioid analgesia. Jomo et al. suggests that there are many possible molecular mechanisms through which estrogen might diminish orphanin FQ (OFQ)-induced antinociception, including decreasing the expression of the (opioid receptor-like-1) ORL1 receptor and/or its coupling to G-proteins which would secondarily modify the affinity of OFQ to the ORL1 receptor [18]. Reciprocally, the requirement for testosterone in mediating the antinociceptive effects of OFQ in the male could be attributable to upregulating expression of the ORL1 gene or enhancing coupling of OFQ receptors to $(\mathrm{Gi} / \mathrm{Go})$ proteins and thus to downstream effectors [18]. Kepler et al. suggests that including gender as an independent variable, morphine does not produce the same degree of antinociception in male and female, especially following the induction of persistent pain [19]. Dahan et al. suggests that animal studies show a tendency for opioids to act more efficaciously in male, human studies are less clear in the presence and direction of any sex effect [20]. According to Palmeira et al., the differences in pain perception related to sex may be associated with hyperalgesia in women but also to the hypoactivity of the inhibitory system of pain in females [21]. In a prospective observational study on 120 patients ( 60 males and 60 females), conducted by Hussain et al., reported that female patients exhibited greater intensity of pain and required higher doses of analgesics compared to males in the immediate post-operative period to achieve a similar degree of analgesia [22].

Endocrine changes during the obesity may be responsible for the difference in the pain threshold. Leptin is a peptide (146 amino acid - long protein encoded by the obesity [ob] gene) secreted by adipose cells that act as a major regulator for food intake and energy homeostasis. Recent studies have shown that leptin, an adipocytokine, played a significant role in nociceptive behavior induced by nerve injury in rats $[23,24]$. Maeda et al. reported that the peripheral effect of leptin on neuropathic pain is mediated via macrophage stimulation [24]. Whereas, central effect of leptin on neuropathic pain is possibly due to the up-regulation of $\mathrm{N}$-methyl-d-aspartate (NMDA) receptors following nerve injury [23]. This could be the reason for more pain in obese rats.

\section{CONCLUSION}

The present study revealed that obese female rats experience more pain sensation to noxious stimuli compared to lean male rats and also the analgesic effect of tramadol is more pronounced in lean male rats compared to obese female rats. Additional research to elucidate the mechanisms driving sex differences and obesity in pain responses is needed to foster future interventions to reduce these disparities in pain and analgesic modulation of tramadol. Henceforth, we are carrying out this study further on molecular level to find out the possible correlation among sex hormones, obesity, pain threshold, and tramadol. Gender and body mass index specific tailoring of pain treatments may become a conceivable outcome in the foreseeable future.

\section{ACKNOWLEDGMENT}

The authors are grateful to Manipal Academy of Higher Education, Manipal, Karnataka, India, for their support toward the accomplishment of this work

\section{AUTHORS' CONTRIBUTIONS}

The first author has designed the study, carried out the experimental part of the work, data compilation, statistical analysis, interpretation of results, and manuscript writing. The second author has guided and monitored the experiment, statistical analysis, interpretation of result, and corrected the manuscript. The third author has coguided the first author.

\section{CONFLICTS OF INTEREST}

All the authors declare that they do not have any conflicts of interest.

\section{REFERENCES}

1. Turk DC, Meichenbaum DH, Berman WH. Application of biofeedback for the regulation of pain: A critical review. Psychol Bull 1979;86:1322-38.

2. Greenspan JD, Craft RM, LeResche L, Arendt-Nielsen L, Berkley KJ, Fillingim RB, et al. Studying sex and gender differences in pain and analgesia: A consensus report. Pain 2007;132:26-45.

3. Riley JL, Robinson ME, Wise EA, Myers CD, Fillingim RB. Sex differences in the perception of noxious experimental stimuli: A metaanalysis. Pain 1998;74:181-7.

4. Rosseland LA, Stubhaug A. Gender is a confounding factor in pain trials: Women report more pain than men after arthroscopic surgery. Pain 2004;112:248-53.

5. Walkers JS, Carmody JJ. Experimental pain in healthy human subjects: Gender differences in nociception and in response to ibuprofen. Anesth Analg 1998;86:1257-62.

6. Finkelstein EA, Fiebelkorn IC, Wang G. State-level estimates of annual medical expenditures attributable to obesity. Obesity Res 2004;12:18-24.

7. World Health Organization. Obesity: Preventing and Managing the Global Epidemic. Geneva (Switzerland): World Health Organization; 1997.

8. Lloret LC, Declèves X, Oppert JM, Basdevant A, Clement K, Bardin C, et al. Pharmacology of morphine in obese patients: Clinical implications. Clin Pharm 2009;48:635-51.

9. McKendall MJ, Haier RJ. Pain sensitivity and obesity. Psychiatry Res 1983;8:119-25.

10. Roane DS, Porter JR. Nociception and opioid-induced analgesia in lean $(\mathrm{Fa} /-)$ and obese (fa/fa) Zucker rats. Physiol Behav 1986;38:215-8.

11. Sirisha G, Rahul PB, Usha NS, Madhu DK. Evaluation of antidepressant effect of chronic administration of tramadol alone and in combination with fluoxetine in low doses in albino mice. Int $\mathrm{J}$ Pharm Pharm Sci 2014;6:101-5

12. Chogtu B, Bairy KL, Satyam SM, Pirasanthan R, Gupta S. Analgesic modulation of tramadol, amitriptyline and gabapentin in male and female wistar rats. Res J Pharm Biol Chem Sci 2013;4:70-8

13. Motaghinejad M, Ebrahimzadeh A, Shabab B. Preventive effect of central administration of venlafaxine on morphine physical dependence, nociception, and blood cortisol level in rat. Int J Prev Med 2014;5:1422-31

14. Prabhu VV, Nalini G, Chidambaranathann N, Kisan SS. Evaluation of anti-inflammatory and analgesic activity of Tridax procumbens linn against formalin, acetic acid and CFA induced pain models. Int J Pharm Pharm Sci 2011;3:126-30.

15. Fillingim RB. Sex-related influences on pain: A review of mechanisms and clinical implications. Rehabil Psychol 2003;48:165-74.

16. Fillingim, RB, Browning $A D$, Powell $T$, Wright RA. Sex differences in perceptual and cardiovascular responses to pain: The influence of a 
perceived ability manipulation. The J Pain 2002;3:439-45.

17. Fillingim RB, Maixner W. Gender differences in the responses to noxious stimuli. Pain Forum 1995;4:209-21.

18. Claiborne J, Nag S, Mokha SS. Activation of opioid receptor like-1 receptor in the spinal cord produces sex-specific antinociception in the rat: Estrogen attenuates antinociception in the female, whereas testosterone is required for the expression of antinociception in the male. J Neurosci 2006;26:13048-53.

19. Kepler KL, Standifer KM, Paul D, Kest B, Pasternak GW, Bodnar RJ. Gender effects and central opioid analgesia. Pain 1991;45:87-94.

20. Dahan A, Kest B, Waxman AR, Sarton E. Sex-specific responses to opiates: Animal and human studies. Anesth Analg 2008;107:83-95.

21. Palmeira CC, Ashmawi HA, Posso IP. Sex and pain perception and analgesia. Rev Bras Anestesiol 2011;61:814-28.

22. Hussain AM, Khan FA, Ahmed A, Chawla T, Azam SI. Effect of gender on pain perception and analgesic consumption in Laparoscopic cholecystectomy: An observational study. J Anaesthesiol Clin Pharmacol 2013;29:337-41.

23. Lim G, Wang S, Zhang Y, Tian Y, Mao J. Spinal leptin contributes to the pathogenesis of neuropathic pain in rodents. J Clin Invest 2009;119:295-304

24. Maeda T, Kiguchi N, Kobayashi Y, Ikuta T, Ozaki M, Kishioka S. Leptin derived from adipocytes in injured peripheral nerves facilitates development of neuropathic pain via macrophage stimulation. Proc Natl Acad Sci 2009;106:13076-81. 